\title{
Focal Epilepsy Secondary to Juxtacortical Lesions in Cerebral Autosomal Dominant Arteriopathy with Subacute Infarcts and Leukoencephalopathy
}

\author{
Tommy L. H. Chan, Manas Sharma, Jorge G. Burneo
}

Keywords: CADASIL, Epilepsy, Seizures, Migraine with aura, Headache

doi:10.1017/cjn.2018.29

Can J Neurol Sci. 2018; 45: 462-463

A 68-year-old man presented with a bilateral tonic-clonic seizure. His medical history included migraine with aura, hypertension and dyslipidemia. His father and mother had ischemic strokes in their fifth decade of life. On initial examination, his vitals were stable, but he had post-ictal confusion, transient right homonymous hemianopsia and mild right-sided Todd's paresis. General systems examinations were unremarkable. MRI of the head showed multifocal confluent white matter lesions (Figures 1A-1B) with a few short association (U) fibers abutting the cortex, notable in the left occipital lobe (Figure 1C). An electroencephalography (EEG) captured a focal seizure originating from the left occipital lobe. Lumbar puncture revealed elevated protein $(864 \mathrm{mg} / \mathrm{L})$ without pleocytosis. Serum metabolic, infective and inflammatory workups were unremarkable. Levetiracetam $20 \mathrm{mg} / \mathrm{kg}$ was loaded intravenously to control his seizures. Subsequent neurological examination demonstrated resolution of his deficits. On further history taking, it was found that the patient had been diagnosed with migraine with aura since the age of 9 years. He described his headache to be bi-frontal, of moderate to severe intensity, throbbing in nature with associated photophobia, phonophobia and nausea. He was experiencing 4 attacks/year with good relief from taking sumatriptan $100 \mathrm{mg}$. In some occasions, his headache would be preceded by a visual aura: he would experience either a gradual- or acute-onset right visual field cut followed by a headache. His aura could last between 1 and 4 hours. Six months before presentation, he started to experience frequent isolated visual auras without headaches.

The diagnosis of cerebral autosomal dominant arteriopathy with subacute infarcts and leukoencephalopathy (CADASIL) was entertained on the basis of the history of migraine with aura, family history of early-onset ischemic strokes and diffuse white matter disease on MRI. On genetic analysis, he was found to be heterozygous for a NOTCH3 mutation, C619C $>\mathrm{T}$, and it is pathogenic for CADASIL. ${ }^{1}$ His atypical visual auras were
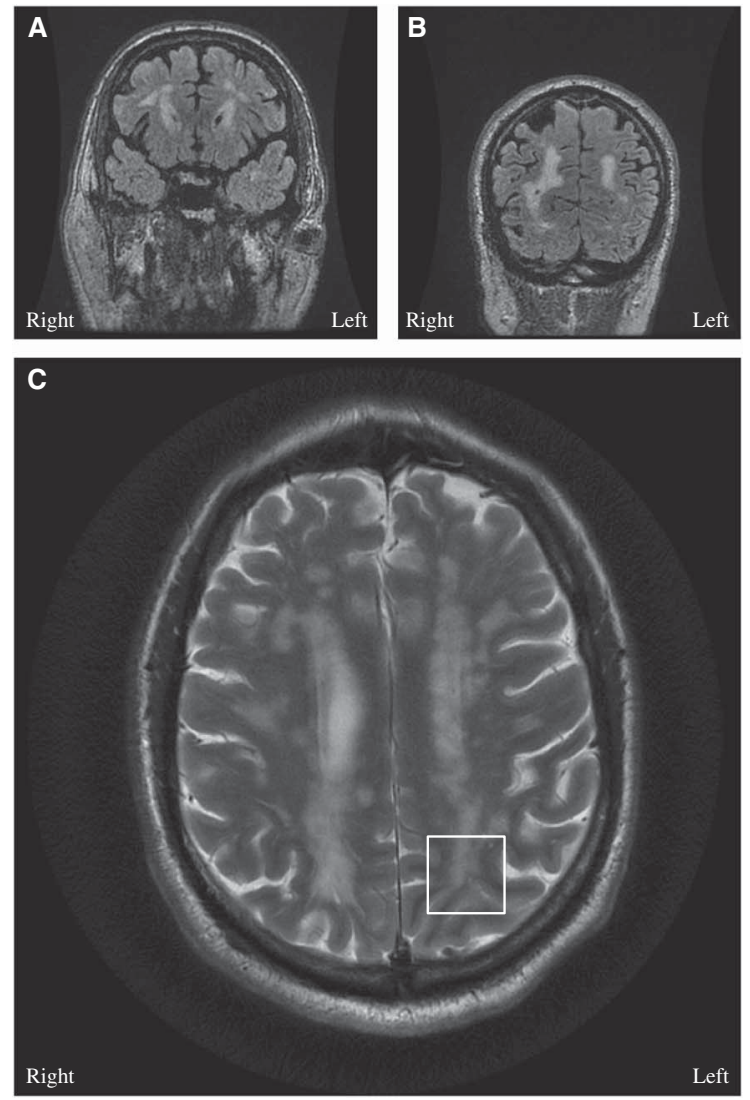

Figure 1: (A, B) Brain MRI coronal T2 Fluid Attenuation Inversion Recovery (FLAIR)-multifocal confluent and discrete subcortical, deep and periventricular lesions are seen involving both cerebral hemispheres, including temporal and occipital lobes. (C) Brain MRI axial T2: few lesions are seen extending through the $U$ fibers and abutting the cortex, notable in the left occipital cortex (white box).

From the Department of Clinical Neurological Sciences, Western University, London, Ontario, Canada (TLHC, JGB); Department of Medical Imaging, Western University, London, Ontario, Canada (MS); Epilepsy Program, Schulich School of Medicine, Western University, London, Ontario, Canada (JGB).

Received February 14, 2018. Final Revisions Submitted March 13, 2018. Date of Acceptance March 14, 2018.


Email: tommy.chan@lhsc.on.ca 
consistent with focal epilepsy originating from the juxtacortical lesions in the left occipital lobe (Figure 1C).

CADASIL is an autosomal dominant inherited angiopathy due to mutations in the NOTCH3 gene on chromosome 19. Migraine with aura is often the initial manifestation of CADASIL, and auras can present with atypical features including acute-onset, long-lasting, isolated motor, sensory, visual or brainstem auras. $^{2}$

Epilepsy is rare in CADASIL, and it has only been reported in $10 \%$ of patients. ${ }^{3,4}$ The postulated pathophysiology is cortical or juxtacortical involvement-that is, subcortical lacunar infarcts secondary to angiopathy at the gray-white matter junction (Figure 1C). ${ }^{3-5}$ In a case series with 102 patients with CADASIL, 10 patients had epileptic seizures and nine of them had a history of $_{\text {strokes. }}^{3}$

Our patient was transitioned to oral levetiracetam at a dose of $500 \mathrm{mg}$ twice daily with no further seizures. He was referred for genetic counseling and his follow-up EEG in 2 months was normal. Lifestyle modifications to decrease stroke risk factors were re-emphasized. Although epilepsy is rare in CADASIL, CADASIL should be on the differential diagnosis in the context of history of migraine with atypical aura, ischemic strokes, family history of early-onset ischemic strokes, diffuse white matter disease and particularly juxtacortical ischemic lesions on MRI.

\section{Disclosures}

TLHC and MS report no disclosures and have no conflicts of interest. JGB has received financial support for research and educational activities from UCB Canada and Eisai, outside the submitted work.

\section{Statement of Authorship}

TLHC examined the patient. All authors wrote and revised the manuscript and created the figures together.

\section{REFERENCES}

1. Oberstein SL, Ferrari MD, Bakker E, et al. Dutch CADASIL Research Group. Diagnostic Notch3 sequence analysis in CADASIL: three new mutations in Dutch patients. Neurology. 1999;52(9):1913.

2. Chabriat H, Joutel A, Dichgans M, Tournier-Lasserve E, Bousser MG. Cadasil. Lancet Neurol. 2009;8(7):643-53.

3. Dichgans M, Mayer M, Uttner I, et al. The phenotypic spectrum of CADASIL: clinical findings in 102 cases. Ann Neurol. 1998; 44(5):731-9.

4. Haan J, Oberstein SA, Ferrari MD. Epilepsy in cerebral autosomal dominant arteriopathy with subcortical infarcts and leukoencephalopathy. Cerebrovasc Dis. 2007;24(2-3):316-7.

5. Bentes C, Pimentel J, Ferro JM. Epileptic seizures following subcortical infarcts. Cerebrovasc Dis. 2001;12(4):331-4. 